\title{
Serum Pleiotrophin Could Be an Early Indicator for Diagnosis and Prognosis of Non-Small Cell Lung Cancer
}

\author{
Zi-Yan Du, Min-Hua Shi, Cheng-Hong Ji, Yong Yu*
}

\begin{abstract}
Aims: Pleiotrophin (PTN), an angiogenic factor, is associated with various types of cancer, including lung cancer. Our aim was to investigate the possibility of using serum PTN as an early indicator regarding disease diagnosis, classification and prognosis, for patients with non-small cell lung cancer (NSCLC). Methods: Significant differences among PTN levels in patients with small cell lung cancer $(S C L C, n=40)$, NSCLC $(n=136)$, and control subjects with benign pulmonary lesions $(\mathrm{n}=21)$, as well as patients with different pathological subtypes of NSCLC were observed. Results: A serum level of PTN of $300.1 \mathrm{ng} / \mathrm{ml}$, was determined as the cutoff value differentiating lung cancer patients and controls, with a sensitivity and specificity of $78.4 \%$ and $66.7 \%$, respectively. Negative correlations between serum PTN level and pathological differentiation level, stage, and survival time were observed in our cohort of patients with NSCLC. In addition, specific elevation of PTN levels in pulmonary tissue in and around NSCLC lesions in comparison to normal pulmonary tissue obtained from the same subjects was also observed $(n=2)$. Conclusion: This study suggests that the serum PTN level of patients with NSCLC could be an early indicator for diagnosis and prognosis. This conclusion should be further assessed in randomized clinical trials.
\end{abstract}

Keywords: Pleiotrophin - non-small cell lung cancer - diagnosis - prognosis

Asian Pac J Cancer Prev, 16 (4), 1421-1425

\section{Introduction}

Pleotrophin (PTN), a secretory growth factor, is also known as heparin affinity regulatory peptide (Laaroubi et al., 1995), heparin binding growth associated molecule (Hampton et al., 1992), heparin binding growth factor-8 (Milner et al., 1989), and heprin binding neutrophic factor (Kovesdi et al., 1990), due to its high affinity to heparin. PTN shares $50 \%$ homogeneous amino acid sequenc with midkine (MK), and these two consist the family of heparin binding growth factors (Kovesdi et al., 1990). PTN is highly consistent among different species, which was reported to present in insects, chicken, mouse, cows, fishes, frogs and human (Tsutsui et al., 1991; Englund et al., 2006).

The overexpression of PTN through either autocrine or pericrine is associated with various metastatic human cancers, including breast, prostatic, ovarian, pancreatic and lung cancer, as well as melanoma, choriocarcinoma, and gliobastoma (Schulte et al., 1996; Jager et al., 1997; Lu et al., 2005). It was reported that PTN promoted cell mitosis, migration and chemotaxis (Kadomatsu et al., 2004). Usually, serum PTN in patients with metastatic tumors were easily detected (Kadomatsu et al., 2004), and were highly consistent with the expression of PTN in tumor tissues (Souttou et al., 1998).
However, clinical studies concerning the overexpression of PTN in lung cancer were limited. In 1997, Jager et al. used RT-PCR to profile the PTN expression in 26 lung cancer cell lines, including 12 small cell lung cancer (SCLC) cell lines and 14 non-small cell lung cancer (NSCLC) cell lines, with normal human bronchial epithelial cells as control. It was revealed that PTN mRNA was expressed in 9 SCLC and 3 NSCLC cell lines (Jager et al., 1997). In a later clinical study with small sample size, the same group reported that $87 \%$ SCLC patients and $63 \%$ NSCLC patients demonstrated elevated serum PTN levels, leading to a 10-fold increase of serum PTN levels in lung cancer patients compared with healthy control subjects, suggesting a potential role could be played by serum PTN levels in early diagnosis and chemo response of lung cancer (Jager et al., 2002). Furthermore, it was demonstrated in our previous studies that knockdown of PTN gene could effectively reduce the transcription and expression of PTN in H446 cells, inhibit cell growth, induce apoptosis and further affect the expression of genes associated with $\mathrm{H} 446$ cells reproduction and migration, using a PTN specific lentivirus RNA interference vector constructed by our group (Yu et al., 2010).

However, the potential role of serum PTN levels in early diagnosis of lung cancer, especially NSCLC, a main type of lung cancer, still requires further investigation. 


\section{Patients and Methods}

\section{Patients and benign control subjects}

Plasma samples were collected from 176 patients (114 male, 62 female, mean age $63.66 \pm 8.68$ years) with histologically confirmed lung cancer registered at department of respiration or thoracic surgery in the second affiliated hospital of Soochow University from 2008-2013, among whom 136 patients were diagnosed with NSCLC (76 cases of squamous cell carcinoma and 60 cases of adenocarcinoma), and 40 others were diagnosed with SCLC. Twenty-two of the NSCLC patients were staged as I, 14 as II, 49 as III and 51 as IV. Also, 42 of the NSCLC patients were classified as low-differentiated (or undifferentiated), 56 as median-differentiated and 38 as highly-differentiated. As for the SCLC patients, 24 cases were classified as limited diseases (LD) and 16 cases as extensive diseases (ED), according to the stage criteria of the veteran's administration lung cancer study group (VALSG). All the lung cancer patients were followed up by telephone every six month. Six patients with NSCLC and 3 patients with SCLC were lost in follow-up. Plasma samples obtained from twenty-one patients with benign pulmonary diseases ( 9 cases of inflammatory pseudotumor, 7 cases of tuberculoma, 3 cases of hamartoma and 2 cases of sarcoidosis) during the same period served as control (12 male, 9 female, mean age $59.90 \pm 18.14$ yrs).

\section{ELISA for PTN serum levels}

Blood samples $(3.5 \mathrm{ml}$ each) from the ulnar vein of patients with either lung cancer or benign pulmonary diseases were collected and stored at $4^{\circ} \mathrm{C}$ prior to ELISA analysis. The samples were centrifuged for $10 \mathrm{~min}$ at 3000 $\mathrm{r} / \mathrm{min}$ at $4^{\circ} \mathrm{C}$ to isolate serum. PTN levels were determined using the human PTN ELISA kit strictly according to the manufactor's manual (Shanghai BlueGene Biotech CO., LTD, Shanghai, China).

RT-PCR and western blot for PTN mRNA and protein expression, respectively
The primer of PTN was designed based on its gene sequence as: 5' -TATGTTCCACAGGTGACATC-3' (sense primer) and 5'-AGAGGACGTTTCCAACTCAA-3' (antisense primer). $\beta$-actin was used as internal control with primer sequences of 5'-AGCGAGCATCCCCCAAAGTT-3' (sense primer) and 5'-GGGCACGAAGGCTCATCATT-3' (anti-sense primer). The amplfication fragments of PTN and $\beta$-actin were 550 and $285 \mathrm{bp}$, respectively. Intratumor specimens, with corresponding peritumor specimens and normal pulmonary tissues (used as control) were resected from surgical subjects suffering squamous cell carcinoma or adenocarcinoma (one of each) with total RNA extracted and reserve transcripted into cDNA using Revertaid First Stand cDNA Syntheis Kit, according to the manufactor's instruction (Fermentas, Canada). The amplified PCR were determined by $1 \%$ agarose gel electrophoresis.

Subsequently, western blot was used to determine the PTN protein levels in the obtained surgical specimens. Protein samples were extracted the homogenized lung specimens with RIPA lysis reagent with the total concentration determined by BCA assay. Protein samples were then separated by $10 \%$ SDS-PAGE gel and transferred to a polyvinylidene fluoride (PVDF) membrane by $2 \mathrm{~h} 300 \mathrm{~mA}$ constant current, followed by saturation with $5 \%$ skim milk powder solution for another 2 h. anti-PTN (1: 100, Abcam, USA) and anti- $\beta$-actin (1: 1000, Santa Cruz Biotechnology, Santa Cruz, CA, USA) primary antibodies were added and incubated overnight at $4^{\circ} \mathrm{C}$. A peroxidase-conjugated secondary antibody (Santa Cruz Biotechnology, Santa Cruz, CA, USA) was added successively and incubated for another hour at room temperature. The protein levels of PTN and $\beta$-actin were then detected using HRP-ECL (Millipore, USA).

\section{Statistical Analysis}

The data obtained was presented as mean \pm standard deviation (SD), with statistical analyses performed with SPSS (version 11.0). Differences with $p<0.05$ were considered statistically significant. The comparisons of mean PTN serum levels among different pathological

Table 1. Summary of Clinco-Patholognical Data and Serum PTN Levels of Recruited Subjects with Statistical Comparisons

\begin{tabular}{|c|c|c|c|c|}
\hline \multicolumn{2}{|l|}{ Group } & Number & $\begin{array}{l}\text { Serum PTN } \\
\text { level }(\mathrm{ng} / \mathrm{ml})\end{array}$ & Statistical comparison \\
\hline \multicolumn{2}{|l|}{ Lung cancer patients } & 176 & $462.51 \pm 184.19$ & \multirow{3}{*}{$\begin{array}{l}\mathrm{F}=13.93, p<0.01, \text { compared with control } \\
\mathrm{t}=1.10, p>0.05\end{array}$} \\
\hline Gender & Male & 114 & $472.90 \pm 177.02$ & \\
\hline Female & 62 & \multicolumn{2}{|l|}{$440.27 \pm 198.53$} & \\
\hline Pathological & squamous cell carcinoma $^{a}$ & 76 & $367.03 \pm 164.68$ & \multirow{3}{*}{$\begin{array}{l}\chi^{2}=105.349, p<0.01 \\
\text { a versus } \mathrm{c}, \chi^{2}=193, p<0.01 \\
\text { b versus } \mathrm{c}, \chi^{2}=118, p<0.01 \\
\text { b versus } \mathrm{c}, \chi^{2}=1826, p<0.05\end{array}$} \\
\hline classification & adenocarcinoma $^{\mathrm{b}}$ & 60 & $423.76 \pm 136.19$ & \\
\hline & Small cell lung cancer ${ }^{\mathrm{c}}$ & 40 & $679.37 \pm 101.78$ & \\
\hline \multicolumn{5}{|l|}{ Non-small cell } \\
\hline \multirow[t]{4}{*}{ lung cancer } & & & 136 & $398.73 \pm 151.51$ \\
\hline & Low $^{d}$ & 42 & $501.77 \pm 122.51$ & $\mathrm{~F}=18.02, p<0.01$ \\
\hline & Medium $^{\mathrm{e}}$ & 56 & $342.08 \pm 144.49$ & $\mathrm{~d}$ versus e, $\mathrm{d}$ versus $\mathrm{f}, p<0.01$ \\
\hline & $\operatorname{High}^{\mathrm{f}}$ & 38 & $368.35 \pm 135.05$ & e versus f, $p>0.05$ \\
\hline \multirow[t]{4}{*}{ Stages } & I & 22 & $168.99 \pm 46.61$ & \multirow[t]{4}{*}{$\chi^{2}=105.35, p<0.01$} \\
\hline & II & 14 & $275.14 \pm 57.27$ & \\
\hline & III & 49 & $386.85 \pm 72.31$ & \\
\hline & IV & 51 & $543.18 \pm 79.21$ & \\
\hline \multicolumn{2}{|l|}{ Control subjects } & 33 & $292.25 \pm 92.52$ & \\
\hline
\end{tabular}




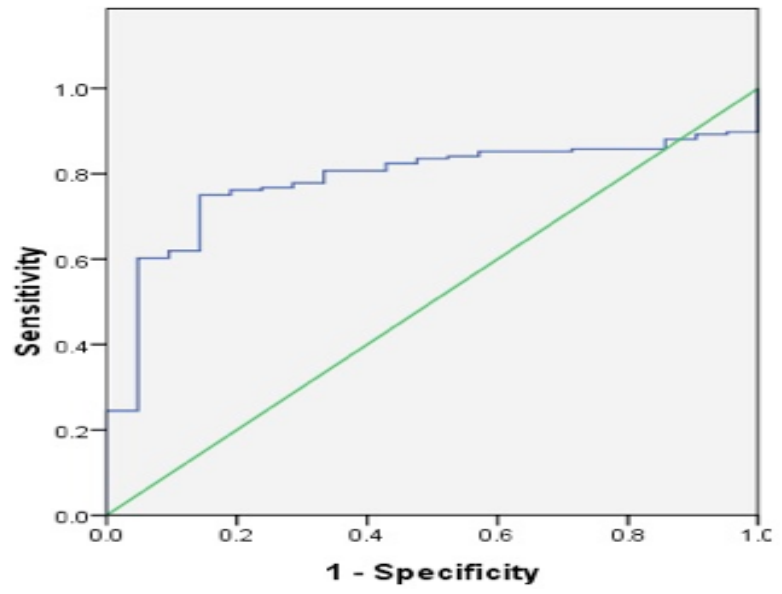

Figure 1. Receiver Operating Characteristic Curve (ROC) Indicating the Sensitivity and Specificity of the Chosed Cutoff in Differentiating Patients with

types/subtypes of lung cancers, genders and stages of SCLC, as well as lung cancers versus benign control were performed with student's test. Receiver operating characteristic curve (ROC) was employed to determine the threshold between the PTN levles of lung cancer patients and benign control subjects. Linear correlation analysis was used to determine the relationship between age and PTN levels. The relationship between differentiation levels and PTN was established by analysis of variance. The correlations between stages of NSCLC and PTN were analyzed using Cruskal-Wallis rank sum test, while the relationship between PTN and different pathological classifications were analyzed by Cruskal-Wallis and Mann-Whitney rank sum test. The survival analysis of both SCLC and NSCLC groups was performed by Cox proportional hazards regression with Enter method for variable selection.

\section{Results}

Elevated PTN serum levels in both SCLC and NSCLC patients

As listed in Table 1, the overall average serum PTN levels of patients with lung cancer was $462.51 \pm 184.19 \mathrm{ng} /$ $\mathrm{ml}$, which was significant higher than that of patients with benign pulmonary diseases $(292.25 \pm 92.52 \mathrm{ng} / \mathrm{ml}), p<0.01$. As shown in Figure 1, serum PTN level of $300.12 \mathrm{ng} / \mathrm{ml}$ was determined as a cutoff value between lung cancer patients and controls, with a sensitivity and specificity of $78.4 \%$ and $66.7 \%$, respectively, using ROC approach. Serum PTN levels of NSCLC patients were determined to be $398.73 \pm 151.51 \mathrm{ng} / \mathrm{ml}$, which was significantly lower than that of SCLC patients $(679.37 \pm 101.78 \mathrm{ng} / \mathrm{ml})$, $p<0.01$, and consistent with previous reports. No apparent correlation of serum PTN levels in lung cancer subjects with either ages $(r=0.075, p>0.05)$ or genders $(p>0.05)$ of patients were observed.

Correlations of elevated PTN serum levels with pathological subtypes, differiention grades and TNM stages in NSCLC patients

Besides the significantly different average PTN a

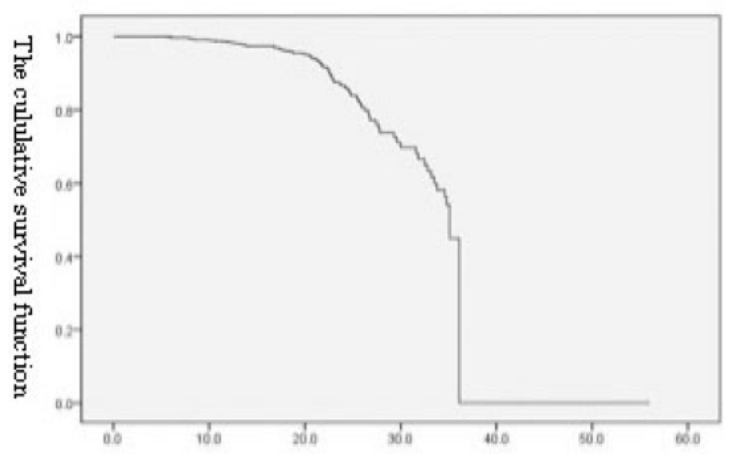

b

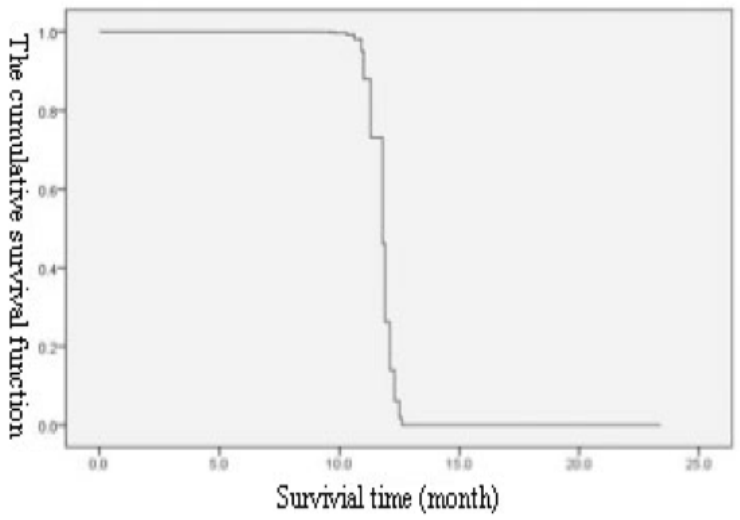

Figure 2. Survival Function at the Mean of Covariates: (a) Non-small cell lung cancer patients; (b) Small Cell Lung Cancer Patients

serum levels observed between NSCLC and SCLC patients, futher classification of NSCLC into squamous cell carcinoma and adenocarcinoma revealed that serum PTN levels significantly varied among different subtypes $(p<0.05)$. Furthermore, correlations between serum PTN levels and differentiation grades of NSCLC were also demonstrated $(p<0.01)$. The serum PTN levels of low, medium and high differentiation group were $501.77 \pm 122.51,342.08 \pm 144.49$ and $368.35 \pm 135.05 \mathrm{ng} / \mathrm{ml}$, respectively, with significant differences spotted between low and medium/high differentiation groups $(p<0.01)$. Moreover, Significantly different serum PTN levels were observed for NSCLC patients at different TNM stages $(p<0.01)$. Similarly, significantly differences were also observed between LD and ED groups of SCLC $(p<0.01)$, which were consistant with literature reports (Jager et al., 2002).

Correlations of elevated PTN serum levels with survival times in both NSCLC and SCLC patients

Cox proportional harzard regression analysis revealed that PTN serum level was an independent risk factor associated with the survival times of NSCLC patients (Figure 2a). The relative risk (RR) value of serum PTN was 1.006 , suggesting that the relative risk of survival time decreasing in NSCLC patients was $1.006 \%$ when serum PTN concentration increased by $1 \%$. As shown in Figure $2 b$, similar correlation was also demonstrated in SCLC patients with the RR value calculated to be 1.051 .

Specific elevation of PTN mRNA and protein levels in intratumor regionsin NSCLC patients

As demonstrated in Figure 3a, the semi-quantitative 


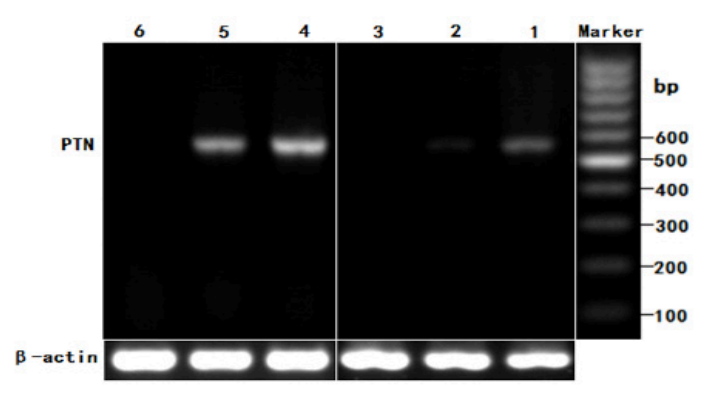

a

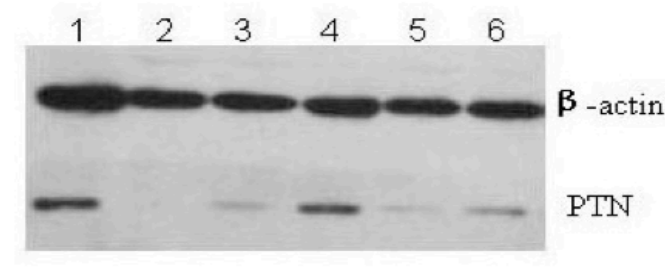

b

Figure 3. Pleiotrophin Expression in Intratumor (1, 4), Peritumor $(3,6)$ and Normal $(2,5)$ Specimens Obtained from a Squamous Cell Carcinoma Patient $(1,2,3)$ and a Ademocarcinoma Patient (4, 5, 6): (a) Pleiotrophin mRNAExpression, Amplification Products of Semi-quantitative RT-PCR Shown in Agarose Gel Electrophoresis; (b) Pleiotrophin Protein Expression by Western Blotting

PCR analysis revealed that there was no apparent PTN mRNA expression in the normal tissues obtained from either squamous cell carcinoma and adenocarcinoma patients. On contrary, mRNA expression levels in the intratumor specimens from both squamous cell carcinoma and adenocarcinoma patients appeared to be highly overexpressed, compared with either normal tissues or peritumor specimen, which also overexpressed PTN mRNA (Figure 3a).

Similar pattern of PTN expression on the protein level was confirmed by western blotting. As shown in Figure $3 \mathrm{~b}$, the intensity of the bands representing the PTN protein levels in intratumor specimens obtained from patients suffering the two subtypes of NSCLC was the highest, followed by peritumor specimens and normal tissues. The lack of visible band for normal tissues suggested that normal pulmonary tissues did not expression PTN protein, which was consistent with the RT-PCR results.

\section{Discussion}

Lung cancer is a leading cause of cancer-related mortality throughout the world (Organization et al., 2014). In China, lung cancer related mortality rate increased by 464.84\% in the past three decades (National et al., 2010; Xie et al., 2014), probably due to the dramatic increased rate of cigarette smoking (Zhang et al., 2003), as well as aggravated air pollution (Pruss et al., 2006).

Early diagnosis is very important. And in this area, one consideration is to develop biomarkers that allow early diagnosis possible. Based on previous results, overexpression PTN could be detected in a wide variety of human cancers, and is believed to be proto-oncogenic.
In-vitro and in-vivo experiments confirmed that elevated expression of PTN could increase the malignant grade of tumor cells, whereas knockdown of PTN can effectively inhibit tumor cell growth, migration and invasion (Schulte et al., 1996; Jager et al., 1997; Kadomatsu et al., 2004; Lu et al., 2005). Current strategies of target therapy towards PTN genes and its receptors include interferences of ribozymes and RNA, monoclonal antibody, etc. (Czubayko et al., 1994; Grzelinski et al., 2006; Papadimitriou et al., 2009). Therefore, PTN, as a potential molecular target of cancer therapy, has become a focus of research.

In this study, elevated serum PTN levels both for SCLC and NSCLC patients were confirmed in a relative large scale clinical investigation, especially for NSCLC. A higher serum PTN level in SCLC patients compared with NSCLC ones demonstrated here is consistent with the results reported early (Jager R et al., 2002; Ji et al., 2014). The change of PTN level between lung cancer group and control was much smaller than previously reported, which could be explained by the different nature of control group (subjects with benign pulmonary diseases versus healthy subjects, as well as the large proportion of NSCLC patients in the current study. Furthermore, since patients with benign pulmonary diseases were used as control, instead of healthy subjects, this study provided a much more realistic and plausible criterion to distinguish lung cancer patients from benign diseases such as inflammatory pseudotumor, which might actually be challenging for early diagnosis of lung cancer. Moreover, this is the first study revealed that serum PTN levels correlated with pathological subtypes in NSCLC patients, which was further confirmed in tumor specimens of NSCLC subjects by both RT-PCR and western blot analysis. In addition, the relative large number of NSCLC patents recruited in the current study allowed more detailed correlation analysis between NSCLC patient serum PTN levels and TNM stages/differentiation levels. All the results suggested that serum PTN could serve as an early indicator for NSCLC diagnosis, in terms of both initial disease confirmation and monitoring of disease progression.

By continuously following patient status through telephone interviews, we were able to investigate whether the serum PTN level at diagnosis was an independent risk factor for prognosis of lung cancer patients. The positive correlation between serum PTN levels and survival times for patients with both NSCLC and SCLC demonstrated in this current study suggested that serum PTN levels could serve as an early indicator for diagnosis and prognosis of lung cancer. In conclusion, this study suggests that serum PTN level of patients with NSCLC could be an early indicator for their diagnosis and prognosis. But this conclusion should be confirmed by randomized clinical trials.

\section{Acknowledgements}

This study is supported in part by the Advanced Project for Returned Personnels Studying Abroad or Doctors of the second affiliated hospital of Soochow University (No. SDFEYBS1102) and Sci-tech Development Planning Project of Suzhou (No. SYSD2012087). 


\section{References}

Czubayko F, Riegel AT, Wellstein A (1994). Ribozyme-targeting elucidates a direct role of pleiotrophin in tumor growth. $J$ Biol Chem, 269, 21358-63.

Englund C, Birve A, Falileeva L, et al (2006). Miple1 and miple2 encode a family of MK/PTN homologues in Drosophila melanogaster. Dev Genes Evol, 216, 10-8.

Grzelinski M, Urban-Klein B, Martens T, et al (2006). RNA interference-mediated gene silencing of pleiotrophin through polyethylenimine-complexed small interfering RNAs in vivo exerts antitumoral effects in glioblastoma xenografts. Hum Gene Ther, 17, 751-66.

Hampton BS, Marshak DR, Burgess WH et al (1992). Structural and functional characterization of full-length heparinbinding growth associated molecule. Mol Biol Cell, 3, 85-93.

Jager R, Noll K, Havemann K, et al (1997). Differential expression and biological activity of the heparin-binding growth-associated molecule (HB-GAM) in lung cancer cell lines. Int $J$ Cancer, 73, 537-43.

Jager R, List B, Knabbe C, et al (2002). Serum levels of the angiogenic factor pleiotrophin in relation to disease stage in lung cancer patients. Br J Cancer, 86, 858-63.

Ji ZQ, Huang XE, Wu XY, et al (2014). Safety of Brucea javanica and cantharidin combined with chemotherapy for treatment of NSCLC patients. Asian Pac J Cancer Prev, 15 (20), 8603-5.

Kovesdi I, Fairhurst JL, Kretschmer PJ, et al (1990). Heparinbinding neurotrophic factor (HBNF) and MK, members of a new family of homologous, developmentally regulated proteins. Biochem Biophys Res Commun, 172, 850-4.

Kadomatsu K, Muramatsu T, et al (2004). Midkine and pleiotrophin in neural development and cancer. Cancer Lett, 204, 127-43.

Lu KV, Jong KA, Kim GY, et al (2005). Differential induction of glioblastoma migration and growth by two forms of pleiotrophin. J Biol Chem, 280, 26953-64.

Laaroubi K, Vacherot F, Delbe J, et al (1995). Biochemical and mitogenic properties of the heparin-binding growth factor HARP. Progress in growth factor research, 6, 25-34.

Milner PG, Li YS, Hoffman RM, et al (1989). A novel 17 kD heparin-binding growth factor (HBGF-8) in bovine uterus: purification and $\mathrm{N}$-terminal amino acid sequence. Biochem Biophys Res Commun, 165, 1096-103.

National Office for Cancer Prevention and Control NCfCR, Disease Prevention and Control Bureau, Ministry of Health (2010). Chinese Cancer Mortality Report: Third National Retrospect Spot-check of Death-Causation. Beijing, China: People's Medical Publishing House.

Organization WH. Cancer: fact sheet no et al (2014). 297. Available from: http: //www.who.int/mediacentre / factsheets/fs297/ en.

Pruss-Ustun A, Corvalan C et al (2006). Preventing Disease Through Healthy Environments. In: Organization WH, editor. Geneva, Switzerland.

Papadimitriou E, Mikelis C, Lampropoulou E, et al (2009). Roles of pleiotrophin in tumor growth and angiogenesis. Eur Cytokine Netw, 20, 180-90.

Schulte AM, Lai S, Kurtz A, et al (1996). Human trophoblast and choriocarcinoma expression of the growth factor pleiotrophin attributable to germ-line insertion of an endogenous retrovirus. Proc Natl Acad Sci USA, 93, 1475964.

Souttou B, Juhl H, Hackenbruck J, et al (1998). Relationship between serum concentrations of the growth factor pleiotrophin and pleiotrophin-positive tumors. J Natl Cancer Inst, 90, 1468-73.
Tsutsui J, Uehara K, Kadomatsu K, et al (1991). A new family of heparin-binding factors: strong conservation of midkine (MK) sequences between the human and the mouse. Biochem Biophys Res Commun, 176, 792-7.

Xie NN, Hu L, Li TH (2014). Lung cancer risk prediction method based on feature selection and artificial neural network. Asian Pac J Cancer Prev, 15 (23), 10539-42.

Yu Y, Shi M-H, Xu X, et al (2010). Construction of pleiotrophin specific lentivirus RNA vector and it effect on the growth and apoptosis of human small cell lung cancer cell line H446. Zhonghua Jie He He Hu Xi Za Zhi, 33, 289-94.

Yu Y, Shi M-H, Xu X, et al (2010). Effects of pleiotrophin gene silencing on the tumor growth related gene expression in human small cell lung cancer cell line H466. Zhonghua Zhong Liu Za Zhi, 32, 405-9.

Zhang H, Cai B et al (2003). The impact of tobacco on lung health in China. Respirology, 8, 17-21. 